\title{
On 3D Reconstruction of Coronal Mass Ejections: I. Method Description and Application to SECCHI-COR Data
}

\author{
M. Mierla • B. Inhester • C. Marqué • L. Rodriguez • \\ S. Gissot • A.N. Zhukov • D. Berghmans · J. Davila
}

Received: 8 December 2008 / Accepted: 17 July 2009 / Published online: 2 October 2009

(C) The Author(s) 2009. This article is published with open access at Springerlink.com

\begin{abstract}
The data from SECCHI-COR1 and SECCHI-COR2 coronagraphs onboard the STEREO mission, which was launched in October 2006, provide us with the first-ever stereoscopic images of the Sun's corona. These observations were found to be useful in inferring the three-dimensional structure of coronal mass ejections (CMEs) and their propagation direction in space. We apply four methods for reconstructing CMEs: $i$ ) Forward modeling technique; ii) Local correlation tracking (to identify the same feature in COR Ahead and COR Behind images) plus tie-point reconstruction technique; iii) Center of mass of the structures in a given epipolar plane plus tie-point reconstruction technique; $i v$ ) Polarization ratio technique. The four techniques are applied to three structured CMEs observed by COR1 and COR2 instruments, respectively, on 15 May 2007, 31 August 2007, and 25 March 2008. A comparison of the results obtained from the application of the four reconstruction algorithms is presented and discussed.
\end{abstract}

STEREO Science Results of Solar Minimum Guest Editors: Eric R. Christian, Michael L. Kaiser, Therese A. Kucera, O.C. St. Cyr

M. Mierla ( $\varangle)$ · C. Marqué · L. Rodriguez · S. Gissot · A.N. Zhukov · D. Berghmans Solar Terrestrial Center of Excellence-SIDC, Royal Observatory of Belgium, Ringlaan 3, 1180 Brussels, Belgium

e-mail: marilena@oma.be

M. Mierla

Astronomical Institute of the Romanian Academy, Str. Cutitul de Argint 5, 040557 Bucharest, Romania

B. Inhester

Max-Planck-Institut für Sonnensystemforschung, Max-Planck Str. 2, 37191 Katlenburg-Lindau, Germany

e-mail: binhest@mps.mpg.de

A.N. Zhukov

Skobeltsyn Institute of Nuclear Physics, Moscow State University, 119992 Moscow, Russia

J. Davila

NASA - Goddard Space Flight Center, Greenbelt, MD 20771, USA

e-mail: josephmdavila@gmail.com 
Keywords Corona, structures · Coronal mass ejections, initiation and propagation

\section{Introduction}

Coronal mass ejections (CMEs) are enormous eruptions of plasma ejected from the Sun into interplanetary space, over the course of minutes to hours. They are crucial for space weather, since they can produce severe magnetic storms when they interact with the Earth's magnetosphere (see, e.g., Schwenn et al., 2006). It is thus very important to infer their true direction of propagation and their three-dimensional (3D) configuration. CMEs are typically observed in white light images provided by coronagraphs (e.g., Large Angle and Spectrometric Coronagraph (LASCO) coronagraphs (Brueckner et al., 1995) onboard the Solar and Heliospheric Observatory ( $\mathrm{SOHO}$ ) spacecraft). The coronagraphs provide us with a particular view of a CME projected on the plane of the sky (POS). From the LASCO images alone, it is not possible to infer the 3D structure of CMEs. The new data from the Solar TErrestrial RElations Observatory (STEREO) (Kaiser et al., 2008), which was launched in October 2006, provide us with the first-ever stereoscopic images of the Sun's atmosphere. The two STEREO spacecraft A and B orbit the Sun at approximately 1 AU near the ecliptic plane with a separation angle between them increasing at a rate of about $45 \%$ year. The stereoscopic images obtained by the Sun Earth Connection Coronal and Heliospheric Investigation (SECCHI) (Howard et al., 2008) aboard STEREO help us to determine the location and the geometry of CMEs in the 3D space.

Several techniques have been developed to infer the location of coronal structures in the 3D space (Pizzo and Biesecker, 2004; Inhester, 2006; Feng et al., 2007; Aschwanden et al., 2008; Howard and Tappin, 2008). Geometric parameters of halo CMEs have been previously measured by (Zhao, Plunkett, and Liu, 2002; Michalek, Gopalswamy, and Yashiro, 2003; Xie, Ofman, and Lawrence, 2004; Krall et al., 2006; Michalek, 2006) using a cone model technique applied on LASCO data. Their assumption is based on the fact that CMEs propagate almost radially beyond $2 R_{\odot}$ with nearly constant angular widths. In another study, Cremades and Bothmer (2004) derived the geometrical properties of structured CMEs from a set of 124 flux rope CMEs observed in LASCO-C2 data. Based on this study, Thernisien, Howard, and Vourlidas (2006) developed a forward-modeling technique for flux-rope like CMEs in order to reproduce the CME morphology. Another technique that has been used to infer the three-dimensional structure of a $\mathrm{CME}$ makes use of polarization measurements of white-light corona (Moran and Davila, 2004; Dere, Wang, and Howard, 2005). With the launch of STEREO spacecraft, several techniques are now being evaluated to derive the direction of propagation of CMEs and as a consequence their propagation speeds in a 3D coordinate system (e.g., Mierla et al., 2008).

In what follows, we apply some of the above mentioned techniques to the SECCHI-COR data, in order to reconstruct the CMEs and/or their directions of propagation: the Polarization ratio (PR) technique (see, e.g., Moran and Davila, 2004) and the Forward modeling (FM) technique (see Thernisien, Howard, and Vourlidas, 2006). Additionally, we make use of the local correlation tracking (LCT) (see, e.g., Trucco and Verri, 1998) to identify the same feature in COR Ahead and COR Behind images. Then, using tie-point (TP) reconstruction method we infer the 3D structure of CMEs. We refer to this as the LCT-TP reconstruction technique. The last method consists in finding the center of mass (CM) of a structure in epipolar planes from their projections onto epipolar lines in the images. Then we apply tie-point reconstruction to get the point coordinates in $3 \mathrm{D}$. We refer to this technique as CM-TP. The four techniques and the acronyms that refer to them are listed in Table 1 . These 
Table 1 Reconstruction techniques.

\begin{tabular}{lll}
\hline Acronym & Name of the technique & Major references \\
\hline FM & $\begin{array}{l}\text { Forward modeling } \\
\text { Local correlation tracking } \\
\text { LCT-TP }\end{array}$ & Thernisien, Howard, and Vourlidas (2006) \\
& $\begin{array}{l}\text { Center of mass } \\
\text { plus tie-point reconstruction }\end{array}$ & Trucco and Verri (1998) \\
CM-TP & Polarization ratio & \\
PR & & Moran and Davila (2004) \\
\hline
\end{tabular}

techniques are applied on three structured CMEs observed by COR 1 and COR2 instruments on 15 May 2007, 31 August 2007, and 25 March 2008. By structured CME we mean the classical 'three-part' CMEs, consisting of a bright, circular front, followed by a dark cavity and a bright compact core (Illing and Hundhausen, 1986). We will compare the results of the different approaches and discuss their success (or failure). This is the first study, to our knowledge, that uses different reconstruction techniques with SECCHI/COR data in order to infer the 3D configuration of CMEs and their direction of propagation.

In this work we will give the fundamentals of each technique and show that they can be applied to the data in a sensible way. There are still several points that can be improved in the application of each technique. Possible improvements, together with a quantitative comparison of the results obtained from the different methods will be studied in a future paper.

\section{Instruments and Observations}

The SECCHI experiment (Howard et al., 2008) on the STEREO mission is a suite of remote-sensing instruments consisting of an extreme ultraviolet imager (EUVI), two white light coronagraphs (COR1 and COR2) and two heliospheric imagers (HI1 and HI2). The SECCHI-COR1 coronagraph is a classic Lyot internally-occulted coronagraph which observes the white light corona from 1.4 to $4 R_{\odot}$ (Thompson et al., 2003). The SECCHI-COR2 is an externally occulted Lyot coronagraph which observes the coronal emission in visible light, having a field of view from 2.5 to $15 R_{\odot}$. The COR 1 and COR2 coronagraphs include a linear polarizer which is used to suppress scattered light and to extract the polarized brightness signal from the solar corona. The polarized brightness is extracted from three sequential images taken with polarization angles of $0^{\circ}, 120^{\circ}$, and $240^{\circ}$. For this study, we used both polarized brightness (pB) and total brightness (tB) images, taken on 15 May 2007, 31 August 2007, and 25 March 2008. The images used are $1024 \times 1024$ pixels $(2 \times 2$ binned).

\section{Data Processing}

The three events we are studying are structured CMEs observed at position angles (PA) around $70^{\circ}, 250^{\circ}$, and $100^{\circ}$, on 15 May 2007, 31 August 2007, and 25 March 2008, respectively (see Figure 1). In order to remove the contribution of coronal streamers and visualize the contribution of CMEs, we subtract a minimum intensity image from each panel. This image is created by taking the minimum value in each pixel, over the images of the day when the CME was observed. The final images were rotated in order to align them with the 

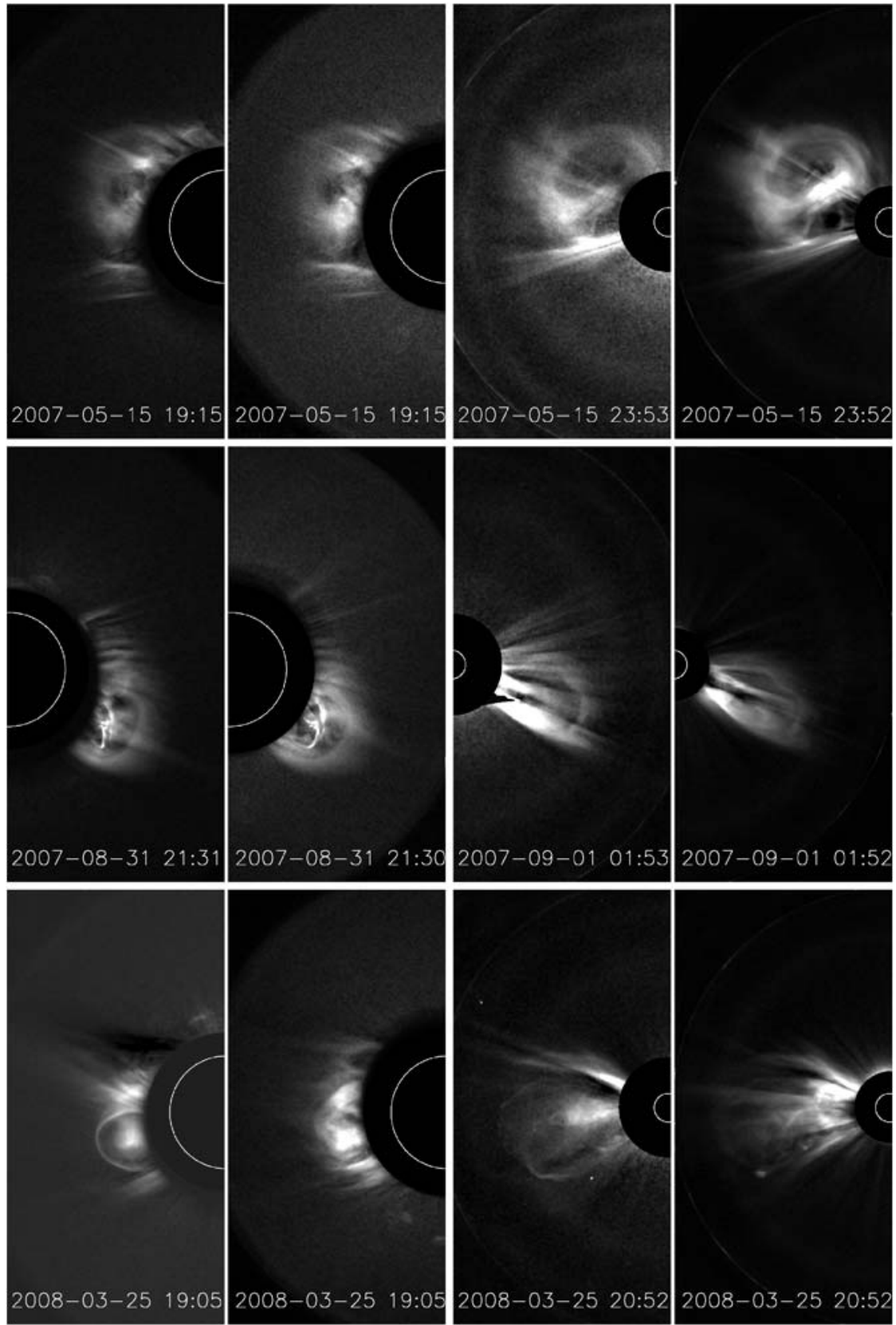

Figure 1 SECCHI-COR images taken on 15 May 2007 (top row), 31 August 2007 (middle row), and 25 March 2008 (bottom row), from both COR1 (the left two columns) and COR2 (the right two columns) coronagraphs. Both A (second and fourth columns) and B (first and third columns) spacecraft images are shown. COR1 images show the white light total brightness corona from 1.4 to $4 R_{\odot}$. COR2 images show the white light total brightness corona from 2.5 to $15 R \odot$. In all panels the visible solar disc is represented by the white circle, while the larger dark disc shows the coronagraph occulter. The images were co-aligned in the STEREO mission plane. 
STEREO mission plane (SMP - the plane which contains the two spacecraft A and B and the center of the Sun). As a consequence, we roll the images so that the SMP north corresponds to the $y$ axis in the image. The images from B are brought to the same Sun center and the same resolution as the A images. To simplify the calculation, we exclude all the other parts of the image where the CME is not seen by selecting a region of interest (ROI). We choose it manually by marking the points on the boundary of the ROI. All the calculations will be done only for the pixels inside the ROI.

\section{Method Description}

The four methods that we use to obtain a three dimensional representation of the CMEs are listed in Table 1 and will be further described below.

The PR and FM methods were designed to be used with only one viewpoint, while for the LCT-TP and CM-TP methods, more than one viewpoint is needed. For the latter two techniques, the views from two vantage points are used to calculate the location of an object in the 3D space, via the tie-point reconstruction. Therefore, a precise identification of the same object in the two images is needed for these methods.

\subsection{Forward Modeling (FM)}

Recent studies (see, e.g., Chen et al., 2000; Vourlidas et al., 2000; Krall and St. Cyr, 2006) have shown that the three-part CMEs and flux rope CMEs represent the same features. Based on the forward model described by Chen et al. (2000) and on a study of Cremades and Bothmer (2004), Thernisien, Howard, and Vourlidas (2006) have developed a forward-modeling technique for flux-rope like CMEs using an empirically defined model of a flux rope, the graduated cylindrical shell (GCS). They have shown that a flux-rope like structure is a good description for three-part CMEs. To compare it with white-light coronagraph observations, Thernisien, Howard, and Vourlidas (2006) have assumed an electron distribution through the GCS and derived synthetic images in total and polarized brightness for various projections of the model using a Thomson scattering ray-tracing program. The program is a numerical implementation of Thomson scattering applied to the solar corona (Billings, 1966). It allows an electron density model to be placed in the 3D space with respect to both the Sun and the observer, and then generates an image of that scene from the observer's location. The same basic technique of reconstructing synthetic Thomson scattering coronagraph images was applied in Chen et al. (2000), although they used a more sophisticated physical model of the flux rope. In this study we have used the CME model geometry proposed by Thernisien, Howard, and Vourlidas (2006). The parameters of the model that are to be fitted to the data are listed in Table 2. The comparison with observations is not quantified but is done by visual inspection. The optimization is done by trial and error.

The GCS is meant to reproduce the large scale structure of flux-rope like CMEs. It therefore consists of a tubular section forming the main body of the structure attached to two cones that correspond to the "legs" of the CME. A sketch of the model is shown in Figure 2.

Note that depending on the separation angle, the two spacecraft may see significantly different leading edges (LEs) of the CME. If the separation angle is small, then the two leading edges are almost the same, otherwise they are quite different and the reconstruction will give a feature in between the real features (see discussion in Section 5.1). 
Table 2 Model parameter description for FM.

\begin{tabular}{|c|c|c|}
\hline Model Parameters & Description & Source \\
\hline Outer shell height & $\begin{array}{l}\text { Maximum distance of the front } \\
\text { side CME surface from the Sun's } \\
\text { center }\end{array}$ & $\begin{array}{l}\text { COR data } \\
\text { if the CME is seen edge on }\end{array}$ \\
\hline $\begin{array}{l}\text { Central propagation } \\
\text { direction }\end{array}$ & Latitude and longitude & $\begin{array}{l}\text { EUVI source region, } \\
\text { if on the visible side of the disk }\end{array}$ \\
\hline Angular width & $\begin{array}{l}\text { Angular width between } \\
\text { the "legs" of the GCS model }\end{array}$ & COR data if the CME is seen head on \\
\hline Aspect ratio & $\begin{array}{l}\text { Ratio of minor torus radius } \\
\text { to the distance from the Sun's } \\
\text { center (self-similar expansion) }\end{array}$ & COR data if the CME is seen edge on \\
\hline Tilt angle of torus & & $\begin{array}{l}\text { Orientation of neutral line } \\
\text { on the solar disk (EUVI, MDI) }\end{array}$ \\
\hline Electron density distribution & & Visual comparison with COR data \\
\hline
\end{tabular}

Figure 2 Sketch of the GCS model. The sphere represents the Sun. The red curve is the leading edge (LE) as seen by the spacecraft $A$ and the blue curve is the leading edge as seen by the spacecraft B.

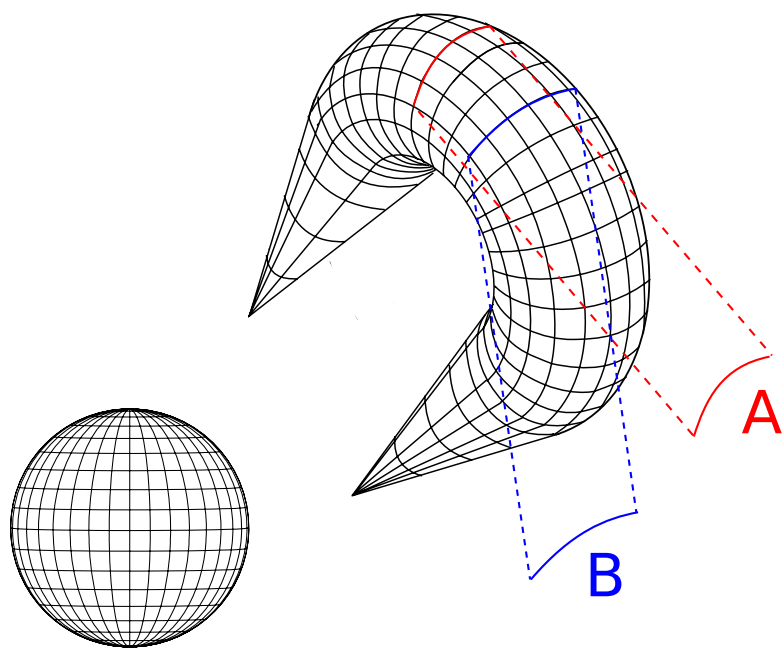

\subsection{Epipolar Geometry and Tie-Point (TP) Reconstruction}

The positions of the two STEREO spacecraft (A for Ahead and B for Behind) and any point in the solar corona to be triangulated define a plane called the epipolar plane (e.g., Inhester, 2006). A special epipolar plane is the STEREO mission plane (SMP) which has as its third point the Sun's center. Hence, all epipolar planes share the two spacecraft positions and the line connecting them (the stereoscopic baseline). Since every epipolar plane is seen head on from both spacecraft, it is reduced to a line in the respective image projections. This line is called epipolar line. Any object identified to be situated on a certain epipolar line in one image must lie on the same epipolar line in the other image. The epipolar lines therefore provide a natural coordinate system for stereoscopic reconstructions. Finding a correspondence between pixels in the images taken by the spacecraft $\mathrm{A}$ and $\mathrm{B}$ is therefore 
reduced to establishing such a correspondence along the same epipolar lines in both images. As a part of the preprocessing, we rectify the images so that their mutual epipolar lines become horizontal. As a simplification of this step, we assume that the epipolar planes are all parallel to the STEREO mission plane. This simplification is justified since distances in the COR fields of view of the order of a few $R_{\odot}$ are much smaller than the distance of $\sim 200 R_{\odot}$ between the Sun and the spacecraft. For the errors introduced by this assumption see Mierla et al. (2008).

Once the correspondence between the pixels is found, the 3D reconstruction is achieved by calculating the lines of sight that belong to the respective pixels in the image and backtracking them into the 3D space. Since the lines of sight (LOS) have to lie in the same epipolar plane, their intersection in this plane is unambiguously defined. This procedure is often called "tie-pointing" (see, e.g., Inhester, 2006). The geometrical reconstruction errors depend on the base angle $\gamma$ between the two STEREO spacecraft. For a point in a given epipolar plane and a pointing error of $\mathrm{d} s$ along the epipolar line in both images, the error in the depth estimate is $\mathrm{d} s / \sin (\gamma / 2)$.

\subsection{Local Correlation Tracking (LCT)}

To find the correspondence between the images, i.e., to identify the same pixels appearing in both images, is one of the key problems of stereoscopic reconstructions (e.g., Inhester, 2006). Unfortunately, CMEs often have a rather diffuse density distribution where prominent, well-located points are difficult to identify. Instead of using a feature-based correspondence, we will use a correlation-based approach (see, e.g., Trucco and Verri, 1998). In such a correlation-based method the elements to be matched are small subimages of fixed size, called match windows. The criterion which decides whether two such windows in each image are positioned on the same object is their mutual correlation coefficient. In order to find correlations, we keep the match window in a fixed position on a given epipolar line in one image and move it along the same epipolar line in the other image. If a maximum of the correlation occurs at a certain shift, the center position of the windows is used for tie-pointing the $3 \mathrm{D}$ region which has probably produced this high correlation. This procedure is subject to some constraints in order to catch meaningful correlations and discard improbable ones. First we use normalized cross-correlations on the match windows $W$ :

$$
\sigma_{\mathrm{AB}}\left(x, x^{\prime}, y\right)=\frac{\int_{W} I_{\mathrm{A}}(x+\xi, y+\zeta) I_{\mathrm{B}}\left(x^{\prime}+\xi, y+\zeta\right) \mathrm{d} \xi \mathrm{d} \zeta}{\left(\int_{W} I_{\mathrm{A}}^{2}(x+\xi, y+\zeta) \mathrm{d} \xi \mathrm{d} \zeta \int_{W} I_{\mathrm{B}}^{2}\left(x^{\prime}+\xi, y+\zeta\right) \mathrm{d} \xi \mathrm{d} \zeta\right)^{1 / 2}}
$$

with $\sigma_{\mathrm{AB}} \in[-1,1]$; where $I_{\mathrm{A}}(x, y)$ and $I_{\mathrm{B}}(x, y)$ are the respective intensities after rectification at positions given by the epipolar line coordinate $y$ and the horizontal coordinate $x$. The integrals are done over the whole pixels in the window $W(\xi$ and $\zeta$ ). This choice is sensitive also to correlations at low intensity levels. In order to discard spurious correlations, we limit the maximum disparity $\left|x-x^{\prime}\right|$. Since the disparity for a given spacecraft configuration is directly proportional to the reconstructed depth, this limitation is equivalent to restricting the reconstructed CME to a certain depth range. The depth is defined as the distance of a feature from the plane of the sky. The shift limits are set so that a depth range of $\pm 5 R_{\odot}$ (corresponding to COR 1 data) and $\pm 12 R_{\odot}$ (corresponding to COR2 data) resulted. Finally, only maxima of $\sigma_{\mathrm{AB}}\left(x, x^{\prime}, y\right)>0.9$ are considered for the tie-point reconstruction.

For this method we are using the total brightness (tB) images because the tB scattering cross section is isotropic within a factor of two. The decrease of the scattering function away from the plane of the sky is gentle and essentially due to the $1 / r^{2}$ decrease of the primary solar radiation. Note that the normalization in correlation (1) takes care of a possibly different signal intensity of a given structure in the two images. 


\subsection{Polarization Ratio Technique (PR)}

It is known that the degree of polarization of Thomson-scattered light by coronal electrons ( $\mathrm{K}$-corona) is a sensitive function of the scattering angle between the incident light direction and the direction towards the observer (Billings, 1966). This effect allows us to estimate an effective scattering angle from the intensity ratio of different polarization brightness which is equivalent to an effective distance of the scatterer from the plane of the sky. Moran and Davila (2004) and Dere, Wang, and Howard (2005) have shown that this effect can be employed to obtain a $3 \mathrm{D}$ reconstruction of a CME from coronagraph images obtained with different polarizer orientations.

Each of the STEREO COR1 and COR2 coronagraphs takes polarized images regularly and we can apply the method independently to the data from both spacecraft. The brightness ratio we use is the ratio of the azimuthal polarized brightness $\mathrm{pB}$ to the unpolarized brightness $\mathrm{uB}(\mathrm{uB}=\mathrm{tB}-\mathrm{pB}$, where $\mathrm{tB}$ is the total brightness). In each case, we subtract a minimum intensity image from the CME images. In this way we get rid of the stable structures, such as streamers. We also ensure that the brightness ratio is not affected by the background due to scattering at dust particles (F-corona) and from the light scattered inside the coronagraph (see, e.g., Moran and Davila, 2004; Dere, Wang, and Howard, 2005). In addition, a $5 \times 5$ median filter is applied to enhance the signal-to-noise ratio of the difference images. At each pixel of an image, the $\mathrm{pB} / \mathrm{uB}$ ratio is calculated and compared with the theoretical value for the Thomson scattering as a function of the scattering angle. Besides the scattering angle, the theoretical brightness ratio depends on the distance of the respective LOS ray from the Sun, on the solar limb darkening, and on the observed wavelength. For the latter two parameters, standard values for the white light are assumed. For the LOS direction of the respective pixel, the scattering angle can then be transformed to an equivalent distance from the plane of the sky. Note that this reconstruction does not give any information about the depth extent of the CME, but rather a weighted mean distance of the CME plasma density along each line of sight. Due to the forward/backward symmetry of Thomson scattering, any scattering angle $\theta \in[0, \pi]$ found allows a second solution of $\pi-\theta$. Consequently, the brightness ratio does not indicate whether the scatterer is in front of or behind the plane of the sky. This ambiguity, however, can be resolved to some extent if it is known whether the CME has been launched at the frontside or the backside of the Sun (e.g., using the EUV observations of the CME source region).

Note that we apply the PR method separately to COR-A and COR-B images. A comparison of the two independent results will give us some estimate about possible errors involved in this method. A discrepancy might arise because the scattering function with distance from the POS for the polarized signal decreases much more rapidly than for the total signal intensity. Since the measured intensities stem from line-of-sight integrals, the polarized and the total intensities may be due to differently weighted contributions along the ray path. Estimates how big this effect may be are difficult, since both the intensities additionally depend on the plasma density distribution along the line-of-sight. If the density varies by orders of magnitude and their enhancements inside the CME are localized, the effect of the scattering function may be small. If the plasma density in a CME is smoothly distributed, the polarized intensity may, due to its more localized scattering function, integrate less plasma than the total brightness signal. This would lead to a smaller $\mathrm{pB} / \mathrm{tB}$ ratio and hence to a distance off the plane of the sky which is larger than what corresponds to the center of gravity of the plasma density distribution. 


\subsection{Center of Mass Reconstruction (CM)}

Unlike the polarized brightness, the total brightness of the Thomson-scattered light varies with the scattering angle as $1+\cos ^{2} \theta$ and can be considered more or less isotropic over the angular range of scattering from the CME to an observer at $1 \mathrm{AU}$. If the isotropy is assumed, then the brightness of a pixel is simply proportional to the integral of the electron column mass density along the respective line-of-sight. The brightness profile integrated along an epipolar line is then proportional to the electron surface mass density in the respective epipolar plane. Moreover, the center of gravity of this integrated brightness profile should be the projection of the center of gravity of the electron surface mass distribution in the epipolar plane. Its determination from two spacecraft A and B allows us to triangulate the center of the CME mass distribution in every epipolar plane it intersects.

We have used the total brightness images for this method. As stated above, the total brightness scatter function off the plane of the sky varies only gently, so we do not expect a large influence. Note that an intensity ratio in the two images does not affect this method because only the positions of the center of mass are in the end compared across the images.

\subsection{Coordinate System and Display of 3D Points}

All our reconstruction results shall be displayed in the same coordinate system for easy comparison. We chose the Heliocentric Earth Equatorial (HEEQ) coordinate system for this purpose. It has its origin at the Sun's center, the $Z$-coordinate axis along the solar rotation axis and the $X$-axis so that Earth lies in the $X-Z$ plane. We are also using Stonyhurst heliographic coordinates which are closely related to HEEQ coordinates (see, e.g., Thompson, 2006). The new coordinates are represented in the spherical coordinate system as latitude, longitude, and distance from the Sun's center. The value of the longitude ranges between $-180^{\circ}$ and $+180^{\circ}$. This also means that the front side disk longitude ranges between $-90^{\circ}$ and $+90^{\circ}$. The image coordinates are given by the $x$-axis (the horizontal axis) and the $y$-axis (the vertical axis).

In order to display the reconstructed points, we use the following procedure. The resulting 3D positions are displayed as isodensity surfaces. In a given geometry, the space around the Sun is divided into a rectangular grid, and the density is calculated for each cell (voxel). The resolution of the reconstruction depends of the cell size. For our data we use a cube of $256 \times 256 \times 256$ cells.

\section{Application of the Methods to the COR Data}

The observations of the three events we are studying in this paper are shown in Figure 1 (COR 1 images in columns 1 and 2 and COR2 images in columns 3 and 4). The separation angle of the two spacecraft was $8^{\circ}$ on 15 May 2007, $28^{\circ}$ on 31 August 2007 and $47^{\circ}$ on 25 March 2008. As we can see from Figure 1 the projected images of A and B spacecraft are quite different for a big separation angle, while for small separation angles the structures are similar in the two images. As a consequence we expect the LCT method to work better for small separation angles. The CMEs on 31 August 2007 and 25 March 2008 were associated with a filament eruption, observed in Extreme UltraViolet Imager (EUVI) $30.4 \mathrm{~nm}$ images. The source region of the CME on 15 May 2007 was an active region at the location N02E47. The orientation of the neutral line was vertical (approximately in the north - south direction) for the events on 15 May 2007 and 31 August 2007. For the 25 March 2008 the neutral line 
Table 3 CME parameters of FM as determined by visual fitting of the GCS model to the observations displayed in Figure 1. The latitude and longitude refer to the HEEQ coordinate system.

\begin{tabular}{|c|c|c|c|c|c|c|}
\hline \multirow[t]{2}{*}{ Date } & \multicolumn{2}{|c|}{ Propagation direction } & \multirow{2}{*}{$\begin{array}{l}\text { Tilt } \\
\text { angle }\end{array}$} & \multirow{2}{*}{$\begin{array}{l}\text { Outer shell } \\
\text { height }\end{array}$} & \multirow{2}{*}{$\begin{array}{l}\text { Aspect } \\
\text { angle }\end{array}$} & \multirow{2}{*}{$\begin{array}{l}\text { Angular } \\
\text { width }\end{array}$} \\
\hline & Long & Lat & & & & \\
\hline 15-05-2007 COR1 & -51 & 7 & 48 & 3.7 & 0.4 & 19 \\
\hline 19:15 UT & & & & & & \\
\hline $\begin{array}{l}\text { 15-05-2007 COR2 } \\
23: 52 \mathrm{UT}\end{array}$ & -49 & 12 & 48 & 11.8 & 0.4 & 27 \\
\hline $\begin{array}{l}\text { 31-08-2007 COR1 } \\
21: 30 \mathrm{UT}\end{array}$ & 63 & -19 & 65 & 3.21 & 0.4 & 17 \\
\hline $\begin{array}{l}\text { 01-09-2007 COR2 } \\
01: 52 \text { UT }\end{array}$ & 65 & -17 & 64 & 11 & 0.35 & 7 \\
\hline $\begin{array}{l}\text { 25-03-2008 COR1 } \\
\text { 19:05 UT }\end{array}$ & -79 & -11 & 0 & 2.8 & 0.48 & 14 \\
\hline $\begin{array}{l}25-03-2008 \text { COR2 } \\
20: 52 \text { UT }\end{array}$ & -78 & -7 & 0 & 12.7 & 0.48 & 14 \\
\hline
\end{tabular}

of the source region was horizontal (east - west orientation). This is reflected in the flux rope model input data (Table 3).

In Section 5.1 we apply the flux rope model to the 31 August CME observed in COR1 images. In Section 5.2 we apply the LCT-TP, PR-TP and PR techniques to the modeled data in order to investigate the reconstruction properties on a known density distribution. In Section 5.3 we apply the reconstruction techniques to the real data recorded by COR 1 on 31 August 2007. The results of all the techniques on the three events we study are discussed in Section 5.4 .

\subsection{Application of the FM to the COR Data}

By fitting the flux rope model of Thernisien, Howard, and Vourlidas (2006) to our data in Figure 1 we obtain the parameter values shown in Table 3. Note that it is mostly the observed shape of the CME front that can be fitted to the projected shape of the model CME. We also modeled the electron density distribution (see Figure 3) but with much less certainty. The fits were made simultaneously to images from COR A and B for one time frame of the sequence for each event. This gave robust estimates for the CME major radius and the latitude of the propagation direction, while its longitude, the angular width and the aspect ratio were less certain, depending on the orientation of the CME with respect to the observation direction. The measurements were made manually several times and by different observers to check for consistency. However, the interpretation of the geometry of one given event (e.g., tilt angle, its extent on the disk, the extent of the CME in the plane of the sky) may be different from one observer to the other, which means that no numerical values are given for error bars. From the parameters of the model we see that the latitude in COR 1 and COR2 images typically differ by about $5^{\circ}$, which we attribute to a deflection of the CME during its propagation. The CMEs on 15 May and 31 August are seen face-on so that the aspect ratio parameter could not be derived from fitting to the data. A value of 0.4 was chosen. The other parameters compare well when derived either from COR1 or COR2 observations. This yields some support for the consistency of the CME model used here. 

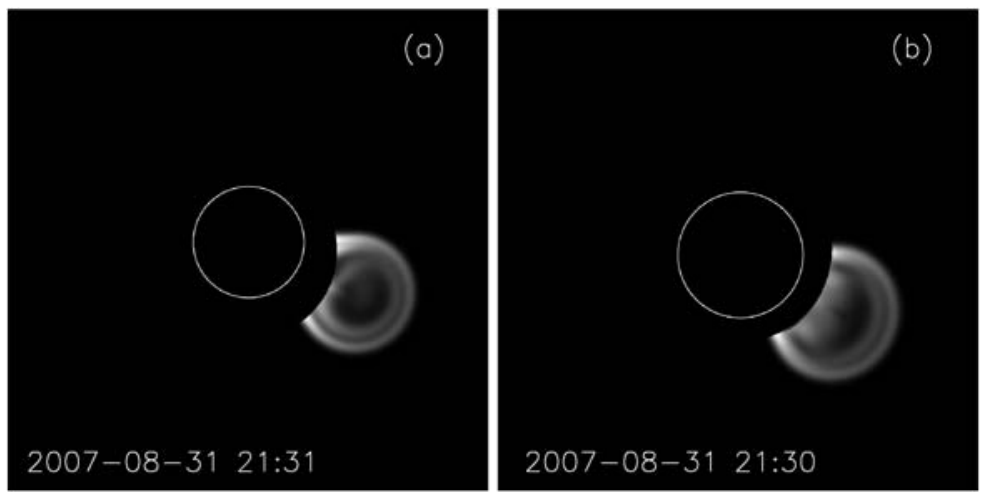

Figure 3 Simulated total brightness images of the GCS model applied to 31 August 2007 CME. Left panel: the GCS model seen from spacecraft B. Right panel: the GCS model seen from spacecraft A.

Figure 3 shows two synthetic coronagraph images of the GCS model applied to the COR1 data on 31 August, viewed from spacecraft A (Figure 3(b)) and spacecraft B (Figure 3(a)). The LE of the CME as seen in the simulated images (Figure 3) corresponds well to the observed LE (Figure 1).

\subsection{Application of LCT-TP, CM-TP and PR to a Model CME}

In order to obtain a more precise idea on the capabilities of the other three methods, we first applied them to synthetic observations that were generated from the GCS model of the CME from 31 August 2007.

After applying the LCT-TP, CM-TP and PR methods to the model data, we obtain the images shown in Figures 4, 5 and 6. In all these representations of our results, we indicate the Sun by the gray sphere. The radius of the outer gridded sphere is $1.5 R_{\odot}$. In a Cartesian HEEQ coordinate system, the CME propagates roughly in the $Y$-direction. The colors represent the distance along the $Y$-axis (blue meaning closer to the Sun center). Two viewpoints were chosen for representation: the left panels of these figures show the CME seen approximately head on ( $X$ and $Z$ in the image plane, $Z$ downwards and $X$ towards right) while the right panels display the CME seen edge on ( $Y$ and $Z$ in the image plane, $Z$ upwards and $Y$ towards right), respectively.

The results for the LCT method are given in Figure 4. For Figures 4(a) and 4(b) the LCT was applied by fixing the window in the image taken by COR1 A and looking for corresponding features in the image taken by COR1 B, while for Figures 4(c) and 4(d) the fixed window was taken in the $\mathrm{B}$ image. The $3 \mathrm{D}$ crest is located in the place where the $\mathrm{CME}$ front side edge is triangulated from each image. The similarity of the two reconstructions demonstrates that the method is reliable. As sketched in Figure 2, a triangulation of the front edge of the CME surface from two vantage points yields a crest that, depending on the surface curvature radius $R_{\text {curv }}$, always lies somewhat ahead of the true CME front surface. This effect should increase with an increasing separation angle $\gamma$ between the spacecraft. The distance between the true and reconstructed leading edges amounts to $R_{\text {curv }}(1-1 / \cos (\gamma / 2))$. For the largest separation angle in our data set $\left(47^{\circ}\right)$, this distance is around $0.1 R_{\text {curv }}$.

Figure 5 shows the reconstructed center of mass of the modeled CME in each epipolar plane it intersects. It therefore gives us mainly information on the longitude of the propagation direction of the CME. 
Figure 4 LCT-TP

reconstruction of the model displayed in Figure 3. Panels (a) and (b) show the reconstruction from A view point and panels (c) and (d) show the reconstruction from B view point. Left panels show the CME seen head on and right panels represent the $\mathrm{CME}$ seen edge on. The numbers on the sphere are the values of HEEQ longitudes. Note that the right panels represent the $\mathrm{CME}$ as seen from the Earth. The images on the left panels were inverted such that solar north is pointing downwards.

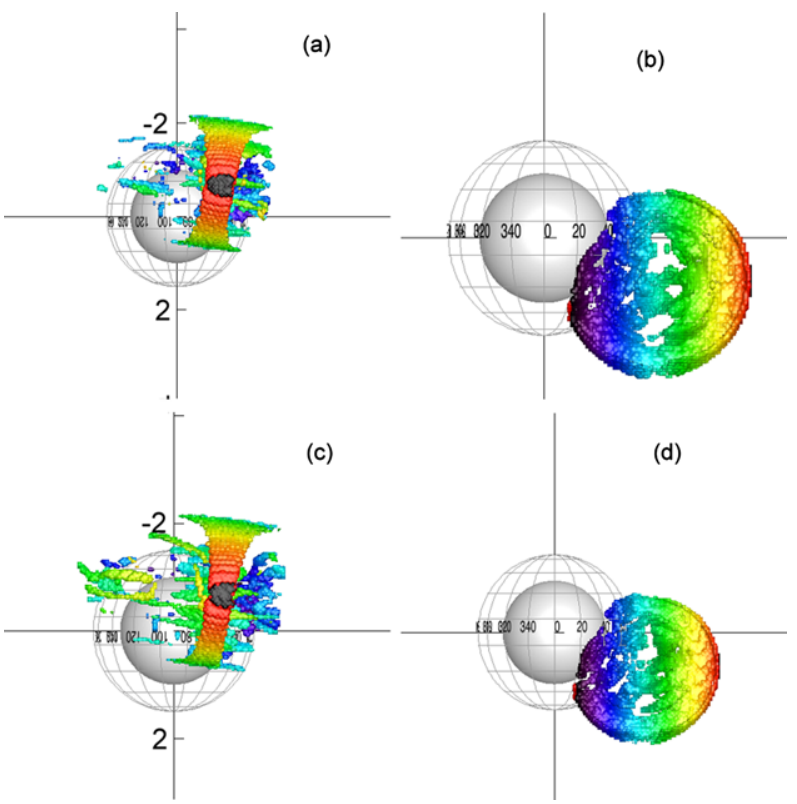

In Figure 6, the upper panels represent the reconstruction by the PR method applied on COR1 A modeled data and the lower panels show the PR reconstruction applied on COR1 $\mathrm{B}$ modeled data. As described in the previous section, this reconstruction does not give any information about the depth extent of the CME, but rather a weighted mean distance of the CME plasma density along each line of sight. The output of the reconstruction is therefore a rough surface which, similarly to the CM-TP method, should be close to the longitudinal center of the CME.

The vector mean of the points from LCT-TP, CM-TP and PR are compared in Table 4. We have weighted the average of the points obtained from the LCT-TP method by the strength of the respective correlation. We see that the direction of this vector mean differs only by a few degrees in latitude and longitude, except for the PR method applied to the modeled B data (PR-B). This may be due to the location of the object compared with the POS of the two satellites (see discussion in Section 5.3). The values also agree well with the parameters for the CME on 31 August 2007 which were used for the model (see Table 3). From the good agreement in the angles we conclude that the techniques can give us a good estimate of the CME direction of propagation. Note, however, that the heights cannot be compared directly because Table 4 gives averages of the CME mass distribution while Table 3 gives the maximum height of the CME front. Even the maximum height of the flux rope center for the 31 August CME derived from Table 3 has a value $3.21 \times(1-0.4 / 2)=2.57$ well beyond the average heights in Table 4.

\subsection{Application of the LCT-TP, CM-TP and PR Methods to the 31 August 2007 CME}

First we apply the LCT-TP method to the COR1 data from 31 August 2007, 21:30 UT (Figure 7). The local correlation is computed in both ways as explained in Section 5.1: first by fixing a window in the image A (Figures 7(a) and 7(b)) and then by fixing the window in the image B (Figures 7(c) and 7(d)). The most clearly reconstructed feature (represented by red points in Figure 7) is the leading edge of the CME. As explained above, due to 
Figure $5 \mathrm{CM}$-TP reconstruction of the modeled CME seen by COR1 on 31 August 2007, 21:31 UT. Panel (a) is the head-on representation and panel (b) is the edge-on representation. The numbers on the sphere are the values of HEEQ longitudes. Note that the right panel represents the $\mathrm{CME}$ as seen from the Earth. The image on the left panel was inverted such that solar north is pointing downwards.

Figure 6 PR reconstruction of the modeled CME seen by COR1 on 31 August 2007, 21:31 UT. The left panels represent the head-on view and the right panels, the edge-on view. The upper panels represent the reconstruction applied on the modeled data as would be seen by the A spacecraft and the lower panels represent the reconstruction applied on the modeled data as would be seen by the B spacecraft. The numbers on the sphere are the values of HEEQ longitudes. Note that the right panels represent the $\mathrm{CME}$ as seen from the Earth. The images on the left panels were inverted such that solar north is pointing downwards.
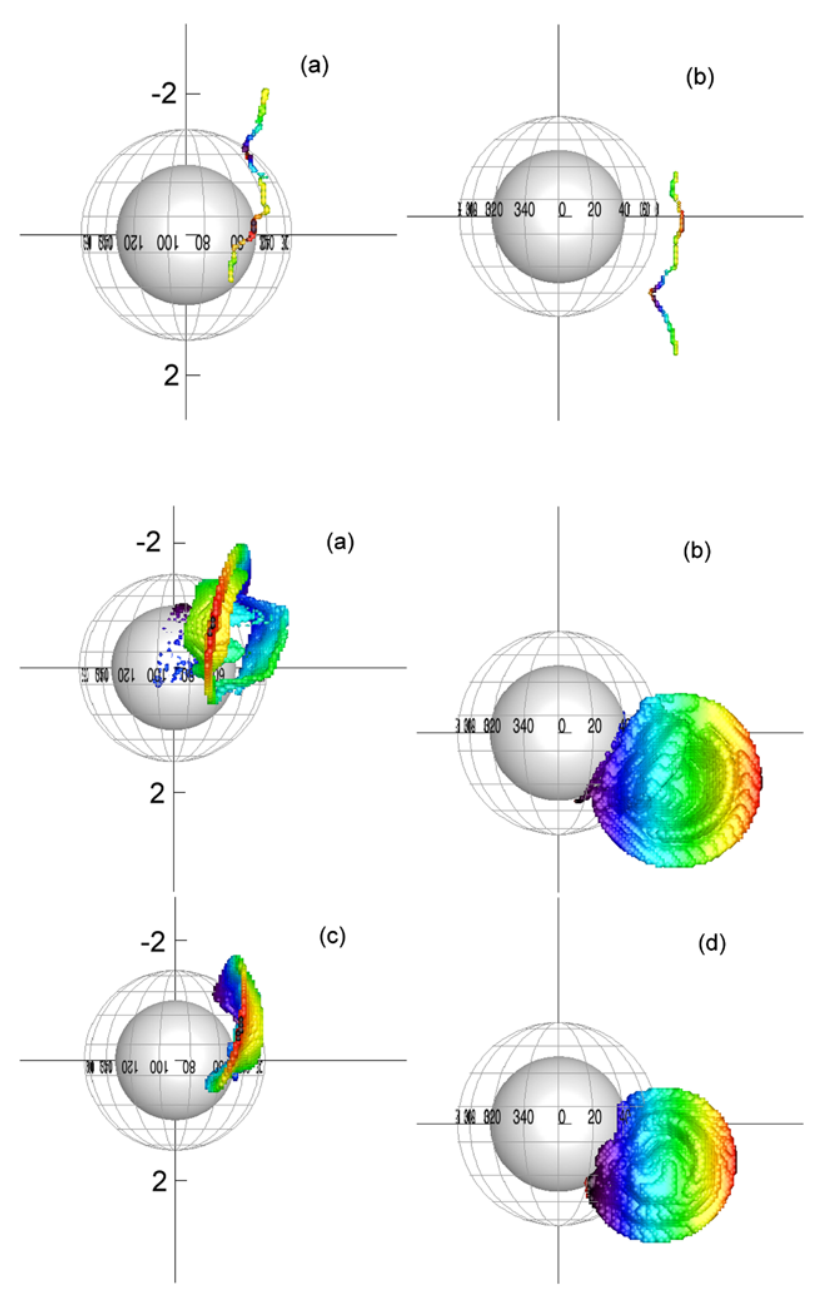

\begin{tabular}{llll}
\hline Method & Longitude & Latitude & Height \\
\hline LCT (from A)-TP & 63 & -19 & 2.22 \\
LCT (from B)-TP & 66 & -19 & 2.22 \\
CM-TP & 61 & -19 & 2.08 \\
PR-A & 64 & -19 & 2.12 \\
PR-B & 54 & -17 & 2.02 \\
\hline
\end{tabular}

Table 4 Mean values of the parameters for points reconstructed using different methods applied on the model COR1 data of 31 August 2007, 21:30UT. The latitude and longitude refer to the HEEQ coordinate system.

the separation angle between the spacecraft of $28^{\circ}$ and a finite curvature of the CME front surface with curvature radius $R_{\text {curv }}$ (see Figure 2), we expect that the reconstructed leading edge lies about $R_{\text {curv }}(1-1 / \cos (\gamma / 2))=0.03 R_{\text {curv }}$ ahead of the true CME front surface. Since $R_{\text {curv }} \sim R_{\text {sun }}$, this seems a negligible deviation in comparison with other reconstruction errors. The LCT results displayed in Figure 7 show some scatter along the line-of-sight (approximately in $X$ direction), best seen from the head-on perspective. As explained in the 
Figure 7 LCT-TP

reconstruction of the $\mathrm{CME}$ on 31 August 2007, COR1 data. Upper panels show the reconstruction from the A view point and lower panels show the reconstruction from the B view point. Left panels show the CME seen head on and right panels represent the CME seen edge on. The numbers on the sphere are the values of HEEQ longitudes. Note that the right panels represent the $\mathrm{CME}$ as seen from the Earth. The images on the left panels were inverted such that solar north is pointing downwards.

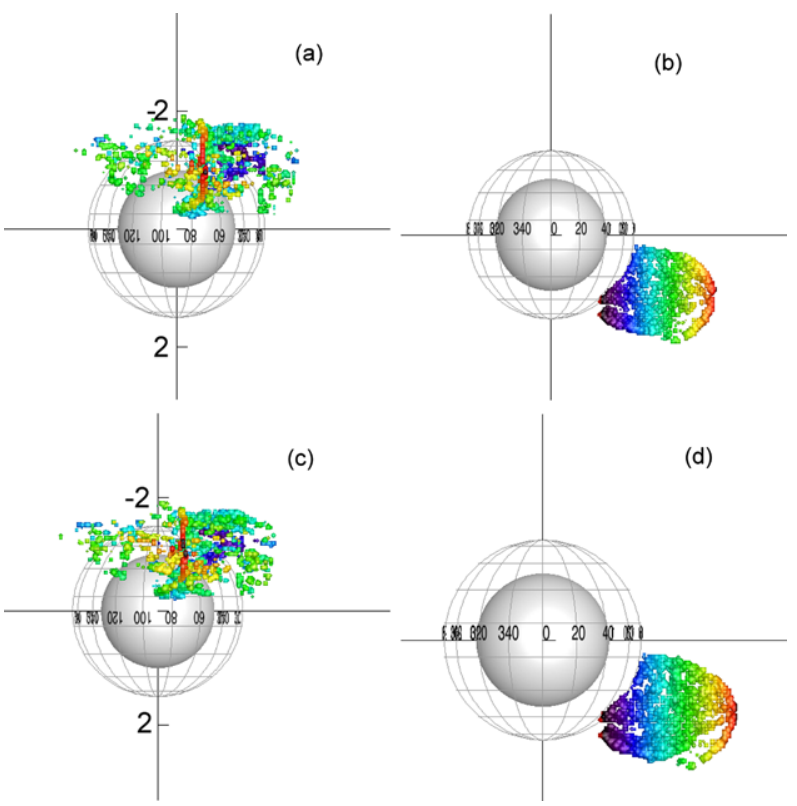

previous chapter, the depth estimates result from the relative shift of the correlation windows for which maxima in the cross-correlation between the images A and B are obtained. If the correlation maxima are due to identical plasma fluctuations inside the CME, then the spread in Figure 7 should indicate the depth extent of the CME. By now, we cannot be sure this is the case. It can be said, however, that the spread we obtained is not limited by the maximum allowed shift in the LCT analysis. This limit would allow for a much wider spread of $\pm 5 R_{\odot}$.

In Figure 8 the reconstruction from the CM-TP method is shown (head on in Figure 8(a) and edge on in Figure 8(b)). The 3D points indicate the direction of propagation of the CME center of gravity in various epipolar planes at that particular time (21:30 UT).

The reconstruction from the PR method applied on COR1 A and B data is shown in Figure 9, upper panels and lower panels respectively. A minimum intensity image was subtracted from the frame with the CME. In this way we get rid of stable structures as F-corona emission and streamers. The results from COR A and B do not agree perfectly. However, they both produce an elongated structure along the LOS (in the positive direction along the $X$-axis) (Figures 9(a) and 9(c)) somewhere near the visible center of the CME. The reason for it is not quite clear, except that a localized area of low $\mathrm{pB} / \mathrm{uB}$ ratio must exist in the COR images inside the CME.

If the $\mathrm{CME}$ is centered at $70^{\circ}$ longitude, its position is $0.21 R_{\odot}$ in front of the POS as seen from STEREO A, and $1.12 R_{\odot}$ in front of the POS as seen from STEREO B. A ratio $\mathrm{pB} / \mathrm{uB}=1.12$ then results for STEREO B from light scattered at the CME center, which represents $33 \%$ of the STEREO A value. For STEREO B this value is particularly low and could possibly be contaminated by polarized light from other off-center regions of the CME. This might explain the differences of up to $40^{\circ}$ in longitude obtained with the other methods.

\subsection{Application of the LCT-TP, CM-TP and PR Methods to the COR Data}

In a first attempt to compare the results quantitatively, we have calculated the vector average of the reconstructed CME using different methods. Table 5 displays the mean values of 
Figure $8 \mathrm{CM}$-TP reconstruction of the CME seen by COR 1 on 31 August 2007, 21:31 UT. Left panel is the CME seen head on and right panel is the CME seen edge on. The numbers on the sphere are the values of HEEQ longitudes. Note that the right panel represents the $\mathrm{CME}$ as seen from the Earth. The image on the left panel was inverted such that solar north is pointing downwards.

Figure 9 PR reconstruction of the CME seen by COR 1 on 31 August 2007, 21:31 UT. The left panels represent the view from the front and the right panels show the view from the side. The upper panels represent the reconstruction applied on COR 1 A data and lower panels represent the reconstruction applied on COR1 B data. The numbers on the sphere are the values of HEEQ longitudes. Note that the right panels represent the CME as seen from the Earth. The images on the left panels were inverted such that solar north is pointing downwards.
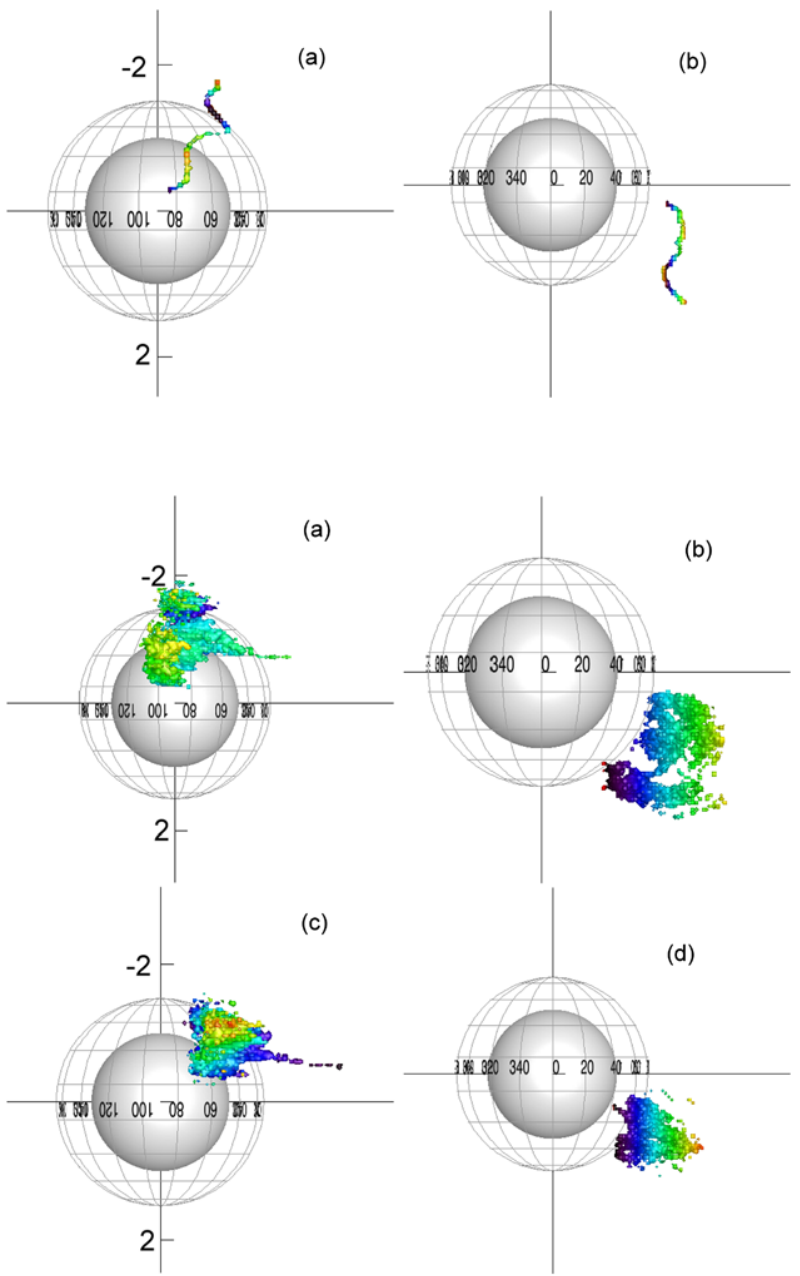

the latitude, longitude, and height. These values for the center of the reconstructed CME should conform with the direction of propagation of the CME. The errors for the longitude in the case of LCT-TP and CM-TP methods are estimated using a pointing error along the epipolar lines of 1 pixel. The depth errors $\left(\right.$ depth $\left._{\mathrm{err}}\right)$ were converted to longitude errors by $\arctan \left(\right.$ depth $_{\text {err }} / R_{\mathrm{CME}}$ ), where $R_{\mathrm{CME}}$ is the distance of the object from the Sun center. The parameters on which the error calculation is based are shown in Table 6. We show also the variation of $\mathrm{pB} / \mathrm{uB}$ depending on the position of an object with respect to the POS as seen from the two spacecraft. From Table 6 we see that at big separation angles an object which is seen in $\mathrm{A}$ is not seen in $\mathrm{B}$ as it is hidden by the occulter.

In general, the three methods (LCT-TP, CM-TP and PR) applied to the same event give approximately the same direction of propagation. The angles agree within $\sim 5^{\circ}$ in latitude and $\sim 10^{\circ}$ in longitude, except for a few outliers produced with the PR method. Compared with the FM method (Table 3) the longitudes of the reconstructed points differ from the longitudes derived from the model in up to $20^{\circ}$ and the latitudes up to $10^{\circ}$. Note however that the propagation direction in this method, while adjusted with the help of the CME 
Table 5 Mean values of the parameters for the points reconstructed using different methods applied to COR1 and COR2 data. The COR1 data were recorded on 15 May 2007, 19:15 UT, 31 August 2007, 21:30 UT, and 25 March 2008, 19:05 UT. The COR2 data were recorded on 15 May 2007, 23:52 UT, 1 September 2007, 01:52 UT, and 25 March 2008, 20:52 UT. The latitude and longitude refer to the HEEQ coordinate system. The errors for LCT-TP and CM-TP methods are estimated using a pointing error along the epipolar lines of 1 pixel.

\begin{tabular}{|c|c|c|c|c|c|}
\hline Date & Instrument & Method & $\begin{array}{l}\text { Mean } \\
\text { Longitude }\end{array}$ & $\begin{array}{l}\text { Mean } \\
\text { Latitude }\end{array}$ & $\begin{array}{l}\text { Mean } \\
\text { Height }\end{array}$ \\
\hline $15-05-2007$ & COR1 & LCT (from A)-TP & $-68 \pm 3$ & 15 & 1.88 \\
\hline $15-05-2007$ & COR1 & LCT (from B)-TP & $-73 \pm 3$ & 15 & 1.8 \\
\hline $15-05-2007$ & COR1 & CM-TP & $-65 \pm 3$ & 7 & 2.27 \\
\hline $15-05-2007$ & COR1 & PR-A & -72 & 8 & 2.05 \\
\hline $15-05-2007$ & COR1 & PR-B & -74 & 10 & 1.98 \\
\hline $15-05-2007$ & $\mathrm{COR} 2$ & LCT (from A)-TP & $-61 \pm 3$ & 11 & 6.97 \\
\hline $15-05-2007$ & $\mathrm{COR} 2$ & LCT (from B)-TP & $-65 \pm 3$ & 13 & 6.78 \\
\hline $15-05-2007$ & $\mathrm{COR} 2$ & CM-TP & $-66 \pm 3$ & 14 & 6.84 \\
\hline $15-05-2007$ & COR2 & PR-A & -53 & 13 & 7.75 \\
\hline $15-05-2007$ & COR2 & PR-B & -64 & 16 & 6.29 \\
\hline 31-08-2007 & COR1 & LCT (from A)-TP & $73 \pm 1$ & -29 & 2.12 \\
\hline 31-08-2007 & COR1 & LCT (from B)-TP & $70 \pm 1$ & -29 & 2.12 \\
\hline 31-08-2007 & COR1 & CM-TP & $72 \pm 1$ & -26 & 2.22 \\
\hline $31-08-2007$ & COR1 & PR-A & 87 & -31 & 1.96 \\
\hline 31-08-2007 & COR1 & PR-B & 59 & -25 & 1.99 \\
\hline 31-08-2007 & COR2 & LCT (from A)-TP & $82 \pm 1$ & -21 & 6.55 \\
\hline 31-08-2007 & COR2 & LCT (from B)-TP & $80 \pm 1$ & -22 & 6.60 \\
\hline 31-08-2007 & COR2 & CM-TP & $75 \pm 1$ & -20 & 6.83 \\
\hline 31-08-2007 & $\mathrm{COR} 2$ & PR-A & 87 & -22 & 6.75 \\
\hline $31-08-2007$ & COR2 & PR-B & 44 & -16 & 7.03 \\
\hline 25-03-2008 & COR1 & LCT (from A)-TP & $-88 \pm 0.5$ & -15 & 1.92 \\
\hline 25-03-2008 & COR1 & LCT (from B)-TP & $-90 \pm 0.5$ & -15 & 1.95 \\
\hline 25-03-2008 & COR1 & CM-TP & $-88 \pm 0.5$ & -14 & 2.06 \\
\hline 25-03-2008 & COR1 & PR-A & -58 & -12 & 1.83 \\
\hline 25-03-2008 & COR1 & PR-B & -97 & -14 & 1.84 \\
\hline 25-03-2008 & $\mathrm{COR} 2$ & LCT (from A)-TP & $-85 \pm 0.5$ & -2 & 6.79 \\
\hline 25-03-2008 & COR2 & LCT (from B)-TP & $-87 \pm 0.5$ & -1 & 6.71 \\
\hline 25-03-2008 & $\mathrm{COR} 2$ & CM-TP & $-92 \pm 0.5$ & -7 & 7.17 \\
\hline 25-03-2008 & COR2 & PR-A & -50 & -11 & 7.03 \\
\hline $25-03-2008$ & COR2 & PR-B & -88 & -7 & 6.91 \\
\hline
\end{tabular}

observations, is mostly determined from the coordinates of the source region on the solar surface. Hence, a deviation from the direction in Table 5 could well be due to a deflection of the CME from its radial direction. The heights in Table 5 are averaged over the $\mathrm{CME}$ and cannot be compared to the heights of the leading edges in Table 3. 
Table 6 Error estimates for the LCT-TP, CM-TP and PR methods. For the PR method the estimates were calculated for an object behind the POS of STEREO A of $0.5 R_{\odot}$, projected distance of $2 R_{\odot}$, east limb. The value of $\mathrm{pB} / \mathrm{uB}$ compared with its maximum value for STEREO A was $69 \%$.

\begin{tabular}{lrlllll}
\hline Date & $\gamma$ & $\begin{array}{l}\text { depth }_{\text {err }} \\
\text { for a COR1 } \\
\text { pixel } \\
\left(R_{\odot}\right)\end{array}$ & $\begin{array}{l}\text { depth } \text { err }_{\text {for a COR2 }} \\
\text { pixel } \\
\left(R_{\odot}\right)\end{array}$ & $\begin{array}{l}\text { CME dist } \\
\text { from POS } \\
\text { STEREO B } \\
\left(R_{\odot}\right)\end{array}$ & $\begin{array}{l}\text { Proj dist } \\
\text { in POS } \\
\text { STEREO B } \\
\left(R_{\odot}\right)\end{array}$ & $\begin{array}{l}\text { Value of pB/uB } \\
\text { compared with } \\
\text { its max value } \\
\text { STEREO B }\end{array}$ \\
\hline $15-05-2007$ & 8 & 0.10 & 0.41 & 0.77 & 1.91 & $49 \%$ \\
$31-08-2007$ & 28 & 0.03 & 0.12 & 1.38 & 1.53 & $23 \%$ \\
$25-03-2008$ & 47 & 0.02 & 0.07 & 1.80 & 0.99 & $15 \%$ \\
\hline
\end{tabular}

\section{Discussion and Conclusions}

The reconstruction of transient CMEs from two STEREO images is an intrinsically undetermined task; an appropriate reconstruction of the 3D electron density would use a tomography-like technique which requires a large number of images of the CME from many different directions. While the reconstruction problem of the full 3D shape of a CME from only two images is strongly underdetermined, it could well be possible that certain parameters can be robustly extracted from the STEREO images or that even more progress can be achieved involving certain well founded a priori assumptions.

As a consequence, we have concentrated in this paper on the determination of the propagation direction of the CME. Firstly, because there is a good chance that the STEREO data is sufficiently sensitive to this parameter; secondly, because the propagation direction of a CME is of considerable importance for space weather predictions.

Even if we are convinced that the directional information is embedded in the CME data from both STEREO spacecraft, it is not obvious how to extract it. We have therefore tried three completely different approaches to reconstruct the CMEs and to determine their propagation angles. In this first paper, we described the fundamentals of each of these techniques and showed that they can be applied to the data in a sensible way. As a reference to our reconstruction results, we used a forward model chosen from a family of flux-rope shapes for the 3D structure of the CME. The parameters of the model were adjusted such that both data from A and B spacecraft were fitted.

Even though the methods differ considerably, we obtained a reasonable agreement in the propagation directions for all three CME cases we investigated. Typical deviations of the propagation angles obtained from the different methods were $5^{\circ}$ in latitude and $10^{\circ}$ in longitude if the PR results are excluded. Somewhat unexpectedly, the LCT method based on the correlation of plasma fluctuations across the two STEREO images did not show a decrease in performance at larger spacecraft separation angles. The reason may be that the less reliable disparity of the correlation maximum at larger separation angles is compensated by a smaller geometrical reconstruction error at these angles. In addition, the LCT-TP gives us some information about the depth of the structure to be reconstructed.

An exception from the narrow scatter of predicted propagation angles was the PR method which in some cases produced outliers off by up to $40^{\circ}$ in longitude. Closer inspection shows that the extraordinarily low polarized emission which gave rise to these outliers originates from the dense plasma of presumably filament material in the core of the CME. An explanation could be that this emission is contaminated by unpolarized $\mathrm{H}_{\alpha}$ radiation from neutral $\mathrm{H}$ in the cool filament material. Another possible reason may be intrinsic to the method: the 
depth estimate along the LOS from the $\mathrm{pB} / \mathrm{uB}$ ratio replaces the distributed emission along the line-of-sight by a scatterer at a unique distance off the plane of the sky. If the CME has a large distance off the plane of the sky, the line-of-sight integral of the $\mathrm{pB}$ and $\mathrm{tB}$ intensities may originate from different sources and the $\mathrm{pB} / \mathrm{uB}$ ratio may fail to yield the correct distance. Moreover, the sign of this distance off the plane of the sky cannot be determined from the method if applied to data from a single spacecraft alone. This ambiguity could perhaps be removed by the observations from two spacecraft at different view angles.

The propagation directions that we found differed by up to $20^{\circ}$ in longitude from the longitude of the source on the solar surface for the same event. The longitudes obtained from COR1 data at a typical CME height of $2 R_{\odot}$ and from COR2 obtained at a height of $7 R_{\odot}$ were at most $10^{\circ}$ apart. We therefore expect that the deflection of the CME from the source longitude is real. Note that the geometrical reconstruction errors were $3^{\circ}$ in the earliest event and decreased with increasing base angle to $0.5^{\circ}$ for the 25 March 2008 observations. It does not seem improbable that a CME may be deflected (see, e.g., St. Cyr et al., 1999) by more than $10^{\circ}$ while still close to the solar surface.

As a conclusion, we might state that with the methods presented here, we are able to determine the most relevant CME propagation direction at the outer boundary of the corona to within $\sim 10^{\circ}$. While the source region coordinates can be determined with better precision, the final CME propagation direction may be deflected in the corona from the radial direction by much more than $10^{\circ}$.

There are still several points that can be improved in the application of each technique. Additional parameters like the depth range of the CME density can be determined. Additional observations from a third spacecraft near Earth could be a valuable help if the STEREO spacecraft attain larger separation angles. Possible improvements, together with a quantitative comparison of the results obtained from the different methods, will be studied in a future paper.

Acknowledgements M.M. would like to thank ROB for the financial support and for the facilities to carry out this work. The authors thank to A. Thernisien for providing the model data and for the productive discussions. We also thank W. Thompson, M. Kramar, N. Srivastava and G. Stenborg for fruitful discussions. We acknowledge the SECCHI/STEREO consortium for providing the data. The SECCHI data used here were produced by an international consortium of the Naval Research Laboratory (USA), Lockheed Martin Solar and Astrophysics Lab (USA), NASA Goddard Space Flight Center (USA), Rutherford Appleton Laboratory (UK), University of Birmingham (UK), Max-Planck-Institut for Solar System Research (Germany), Centre Spatiale de Liège (Belgium), Institut d'Optique Théorique et Appliquée (France), Institut d'Astrophysique Spatiale (France). We also thank the anonymous referee for useful comments and suggestions.

Open Access This article is distributed under the terms of the Creative Commons Attribution Noncommercial License which permits any noncommercial use, distribution, and reproduction in any medium, provided the original author(s) and source are credited.

\section{References}

Aschwanden, M.J., Burlaga, L.F., Kaiser, M.L., Ng, C.K., Reames, D.V., Reiner, M.J., Gombosi, T.I., Lugaz, N., Manchester, W., Roussev, I.I., et al.: 2008, Space Sci. Rev. 136, 565.

Billings, D.E.: 1966, A Guide to the Solar Corona, Academic Press, London.

Brueckner, G.E., Howard, R.A., Koomen, M.J., Korendyke, C.M., Michels, D.J., Moses, J.D., Socker, D.G., Dere, K.P., Lamy, P.L., Llebaria, A., et al.: 1995, Solar Phys. 162, 357.

Chen, J., Santoro, R.A., Krall, J., Howard, R.A., Duffin, R., Moses, J.D., Brueckner, G.E., Darnell, J.A., Burkepile, J.T.: 2000, Astrophys. J. 533, 481.

Cremades, H., Bothmer, V.: 2004, Astron. Astrophys. 422, 307.

Dere, K.P., Wang, D., Howard, R.: 2005, Astrophys. J. 620, L119. 
Feng, L., Inhester, B., Solanki, S., Wiegelmann, T., Podlipnik, B., Howard, R.A., Wuelser, J.-P.: 2007, Astrophys. J. 671, L205.

Howard, T.A., Tappin, S.J.: 2008, Solar Phys. 252, 373.

Howard, R.A., Moses, J.D., Vourlidas, A., Newmark, J.S., Socker, D.G., Plunkett, S.P., Korendyke, C.M., Cook, J.W., Hurley, A., Davila, J.M., et al.: 2008, Space Sci. Rev. 136, 67.

Illing, R.M.E., Hundhausen, A.J.: 1986, J. Geophys. Res. 91, 10951.

Inhester, B.: 2006, arXiv:astro-ph/0612649.

Kaiser, M.L., Kucera, T.A., Davila, J.M., St. Cyr, O.C., Guhathakurta, M., Christian, E.: 2008, Space Sci. Rev. 136, 5.

Krall, J., St. Cyr, O.C.: 2006, Astrophys. J. 652, 1740.

Krall, J., Yurchyshyn, V.B., Slinker, S., Skoug, R.M., Chen, J.: 2006, Astrophys. J. 642, 541.

Michalek, G.: 2006, Solar Phys. 237, 101.

Michalek, G., Gopalswamy, N., Yashiro, S.: 2003, Astrophys. J. 584, 472.

Mierla, M., Davila, J., Thompson, W., Inhester, B., Srivastava, N., Kramar, M., St. Cyr, O.C., Stenborg, G., Howard, R.A.: 2008, Solar Phys. 252, 385.

Moran, T.G., Davila, J.: 2004, Science 306, 66.

Pizzo, V.J., Biesecker, D.A.: 2004, Geophys. Res. Lett. 31, 21802.

Schwenn, R., Raymond, J.C., Alexander, D., Ciaravella, A., Gopalswamy, N., Howard, R., Hudson, H., Kaufmann, P., Klassen, A., Maia, D., et al.: 2006, Space Sci. Rev. 123, 127.

St. Cyr, O.C., Burkepile, J.T., Hundhausen, A.J., Lecinski, A.R.: 1999, J. Geophys. Res. 104, 12493.

Thernisien, A.F.R., Howard, R.A., Vourlidas, A.: 2006, Astrophys. J. 652, 763.

Thompson, W.T.: 2006, Astron. Astrophys. 449, 791.

Thompson, W.T., Davila, J.M., Fisher, R.R., Orwig, L.E., Mentzell, J.E., Hetherington, S.E., Derro, R.J., Federline, R.E., Clark, D.C., Chen, P.T., et al.: 2003, In: Keil, S.L., Avakyan, S.V. (eds.) Innovative Telescopes and Instrumentation for Solar Astrophysics, Proc. SPIE 4853, 1.

Trucco, E., Verri, A.: 1998, Introductory Techniques for 3-D Computer Vision, Prentice-Hall, Englewood Cliffs.

Vourlidas, A., Subramanian, P., Dere, K.P., Howard, R.A.: 2000, Astrophys. J. 534, 456.

Xie, H., Ofman, L., Lawrence, G.: 2004, J. Geophys. Res. 109, A03109.

Zhao, X.P., Plunkett, S.P., Liu, W.: 2002, J. Geophys. Res. 107, 1223. 